\title{
The Nonlinear Dynamics Characteristics of Stock Market and Its Variation
}

\author{
Jun Meng, Tianyu Zhu, Xiao Chen, Xiang Yin \\ College of Electrical Engineering, \\ Zhejiang University, \\ Hangzhou, China \\ junmeng@zju.edu.cn
}

\begin{abstract}
The stock market is a kind of complex system with all kinds of interactions. It also shows a nonlinear characteristic. In this paper, we analysed the time series from Dow Jones indexes itself and the time series from its fluctuation difference and extracted its correlation dimension and Lyapunov exponent, which shows a chaotic dynamic characteristic in it. Moreover, we also analysed the variation of chaotic characteristic indexes in long term, and found that the correlation dimension has a quasi-periodical variation and some rapid drops in some specific years. The variation of the correlation dimension can be used to reflect some internal changes in stock market.
\end{abstract}

Keywords-stock market; Dow Jones indexes; correlation dimension; the maximum Lyapunov exponent; phase space reconstructiontt

\section{INTRODUCTION}

The stock market is a kind of complex system with all kinds of interactions. Since M.Stutzer found chaos phenomenon in economy system in $1980^{[1]}$, more and more researchers have studied economy system from this perspective. In, 1988,W.A.Barnett and Chen found a strange attractor with 1.5 dimensions in US money currency index ${ }^{[2]}$. Also, in 1989, Hesieh researched on exchange rate fluctuation by the use of chaos method ${ }^{[3]}$. The studies all showed that there is chaos exist in economy system.

However, even though, any studies have proved the existence of chaos in finance system ${ }^{[4-5]}$ there are still some questions left. Is the time series of exchange fluctuation (DIF) random or chaos? Is the chaotic characteristic stays stable in a exact value or it will ranges with the system evaluation? So, these two aspects are what we mainly want to talk about next.

As we know, a chaotic system is a deterministic nonlinear system and it has the sensitive dependence on initial conditions, that is to say, the subtle changes in initial conditions would lead to a different result in the future. So far, it is generally considered that a Lyapunov exponent which is greater than zero and a fractional dimension are the character of chaotic system. The two are also evidences to judge whether a system is a chaotic system . Also, correlation dimension has a very important meaning for a nonlinear time series when it is reconstructed in the higher dimension space. In this paper, we will use these indexes to reflect the nonlinear characteristics in stock market.

\section{MATHEMATICAL MODELS}

\section{A. Phase Space Reconstruction}

In most of the times, what we observe in an experiment is not a phase space object but a time series. Moreover, from a formal point of view, a time series looks like to be random may contain chaotic motion. However, the chaotic motion exist in at least three dimensional sutonomous dynamical systems. Therefore we have to convert the obervations into a state vectors in higher dimensional space to fully study the characteristic of the time series.

Because, the time series is actually the result from projection in higher dimensional system, so it is possible for us to get profound implications of multi-dimensional space from one-dimensional scalar data. In 1980,Packard and others firstly made up the idea of Phase Space Reconstruction $^{[6]}$. In 1981, according to Packard et al. and Takens $^{[7]}$,the method of time delays can be used to embed a scalar time series. The principle is as follows:

For a time series $\left\{X_{i}\right\}, i=1,2, \ldots n$, we can reconstruct them into an m-dimensional space by set a group of phase space vectors:

$$
\begin{aligned}
& X_{i}=\left\{X_{i}, X_{i+\tau}, \ldots, X_{i+(m-1) \tau}\right\} \\
& \mathrm{i}=1,2,3 \ldots \mathrm{m}, \quad x_{i} \in R^{m} \quad m=n-(m-1) \tau
\end{aligned}
$$

Where $\tau$ is time delay and Takens' theorem point out that if the embedding dimension $m \geq 2 d+1$, $\mathrm{d}$ is the number of independent variables for the system, it will be sufficient to construct a new space such that the attractor in this space is equivalent to the original one.

\section{B. Mutual Information Method to determine time delays}

The idea of MI method was first from Shannon's concept of mutual information between two measurements $a_{i}$ and $b_{i}$ drawn from sets $\mathrm{A}$ and $\mathrm{B}$ of possible measurements. When $P_{A B}(a, b)$ is the joint probability density for $\mathrm{A}$ and $\mathrm{B}, P_{A}(a)$ and $P_{B}(b)$ are the individual probability densities for the measurement of $\mathrm{A}$ and of $\mathrm{B}$, we have the mutual information of them: 


$$
I_{A B}=\sum_{a_{i}, b_{j}} P_{A B}\left(a_{i}, b_{j}\right) \log _{2}\left[\frac{P_{A B}\left(a_{i}, b_{j}\right)}{P_{A}\left(a_{i}\right) P_{B}\left(b_{j}\right)}\right]
$$

The less correlation between them, the more it closes to 0 ; so it serves as a perfect indication of how two measurements correlates to one and another. We can calculate the mutual information between $x(t), x(t+\tau)$, as $I(\tau)$.The minimum point of $I(\tau)$ means the less correlation between $x(t)$ and $x(t+\tau)$, and time delay $\tau$ in this point can be used as the best time delays in the reconstruction.

\section{Correlation Dimension}

In most of the times, dimension is one of the most important characteristics of the attractor. It is an important characteristic to chaotic system that there is a fractal dimension of attractor. For a nonlinear system, fractal dimension can also be used to describe the complexity of the attractor. In 1983, Grassberger \& Procaccia introduced a practical algorithm ${ }^{[8-9]}$ to calculate correlation dimension as follow:

TABLE I. THE STEPS FOR CALCULATING THE CORRELATION DIMENSION WITH GP METHOD

According to the time delay $\tau$ and a embedding dimension $m_{0}$ to reconstruct a phase space
\[ X(i)=\{x(i), x(i+\tau), \ldots, x(i+(m+1) \tau)\},(\mathrm{i}=1,2, \ldots, \mathrm{n}) \]
2.Calculate the correlation integration $C(r)=\frac{2}{N(N-1)} \sum_{i=1}^{N} \sum_{j=i+1}^{N} H\left(r-\left\|X_{i}-X_{j}\right\|\right)$
$H(x)$ is the Heaviside step function, $H(x)=0$ if $x \leq 0$ and $H(x)=1$ for $x>0$.
In the limit of an infinite amount of $\operatorname{data}(N \rightarrow \infty)$ and for small $r$, we expect $\mathrm{C}$ to scale like a power law, as $C(r) \alpha r^{D}$, so we can define the
correlation dimension D by $D=\lim _{r \rightarrow 0} \lim _{N \rightarrow \infty} \frac{\partial \ln C(r, N)}{\partial \ln r}$
3.Choose a larger embedding dimension m and repeat step $1 \& 2$,until the correlation dimension $\mathrm{D}$ is relatively stable. And at this time, $\mathrm{D}(\mathrm{m})$ is
the real correlation dimension and $\mathrm{m}$ is best embedding dimension.

\section{The Largest Lyapunov Exponent}

In a chaotic system, it is impossible to make a long-term forecast. Some tiny change of the initial condition will expand rapidly at an exponential speed. Lyapunov exponent can be used to reflect the speed of the exponential divergence of the track. In 1985, Wolf and others gave a practical method ${ }^{[10]}$ to evaluate the largest Lyapunov exponent based on phase trajectory evolution. The specific method is as follow:

TABLE II. THE STEPS FOR CALCULATING THE LARGEST LYAPUNOV EXPONENT WITH WOLF METHOD

1. Calculate the average cycle P, with FFT

2. Calculate time delay $\tau$ with mutual information method, and calculate embedding dimension m with G-P method;

3. According to the time delay $\tau$ and a embedding dimension m reconstruct a phase space

$$
X(i)=\{x(i), x(i+\tau), \ldots, x(i+(m+1) \tau)\},(\mathrm{i}=1,2, \ldots, \mathrm{n})
$$

4. Set a basis spot $X\left(t_{0}\right)$ and find the nearest spot $X_{0}\left(t_{0}\right)$, and the distance between them is $L_{0}$.After,time evolution of $t_{1}$ later,the distance between them is $L_{0}^{\prime}\left\|X\left(t_{1}\right)-X_{0}\left(t_{1}\right)\right\|>\varepsilon, \varepsilon>0$.

5. Take $X\left(t_{1}\right)$ as the basis spot and repeat step.4 until $\mathrm{X}(\mathrm{t})$ research the end of time series $\mathrm{N}$. M is the total number of the evolution iteration,then the largest Lyapunov exponent is:

$$
\lambda=\frac{1}{t_{M}-t_{0}} \sum_{t=0}^{M} \ln \left(\frac{L_{i}^{\prime}}{L_{i}}\right)
$$




\section{DATA ANALYSIS}

A. The detection of chaos characteristic in Dow Jones indexes

For Dow Jones indexes, we take opening price every day as time series for analysis, and this time series we take contans from Jan, 1999 to May, 2002, as shown in Fig.1. Because at this stage, it was the first time the stock market reached 10,000 points, and it continued to fluctuate around 10,000 points for more than two years. Thus, it is a high possibility for us to find that there is a nonlinear characteristic exist in this time series.

With the mathematical models we present above, we can get that, mutual information point of the first minimum value corresponding to delay time can be used as the best time delay, which number is 7 , as shown in Fig.2.

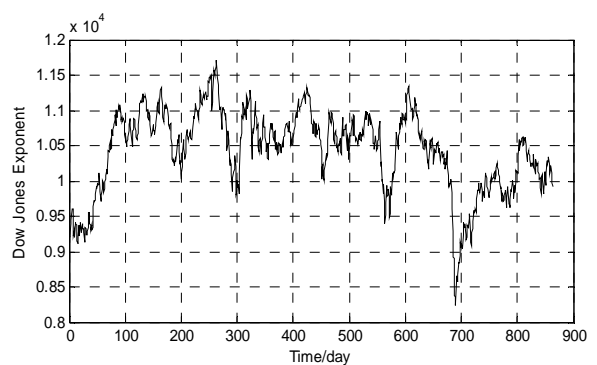

Figure 1. Time seires of Dow Jones from Jan 1999 to May 2005

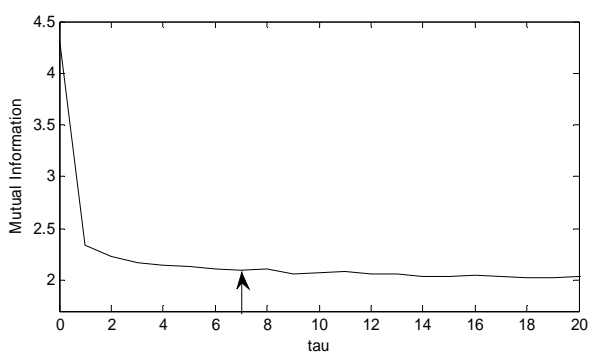

Figure 2. MI method seeking best time delay

With G-P method, it is easy to see that, when the value of $r$ is very small, there is a linear correlation for $\operatorname{lnC}(r)-\ln r$ curve, as shown in Fig.3(a). With the embedding dimension increases, the curve will be in parallel, which means the correlation dimension is saturated, as shown in Table.3 and Fig.3(b). We can determine that the correlation dimension is 4.4 and the best embedding dimension is 8 . With Wolf method, we can calculate the largest Lyapunov exponent is 0.0528 . As the dimension of the attractor is fractional and the largest Lyapunov exponent is positive, we can determine that this time series has chaos character. Also, we can reconstruct phase space with parameters we calculated above, as shown in Fig.4.

TABLE III. DIFFERENT CORRELATION DIMENSION D(M) WITH DIFFERENT M, $\tau=7$

\begin{tabular}{|c|c|c|c|c|c|c|c|c|c|c|}
\hline Embedding Dimension m & 2 & 3 & 4 & 5 & 6 & 7 & 8 & 9 & 10 & 11 \\
\hline $\mathbf{D}(\mathbf{m})$ & 0.978 & 1.911 & 3.302 & 3.594 & 4.582 & 4.645 & 4.140 & 4.044 & 4.258 & 3.984 \\
\hline
\end{tabular}

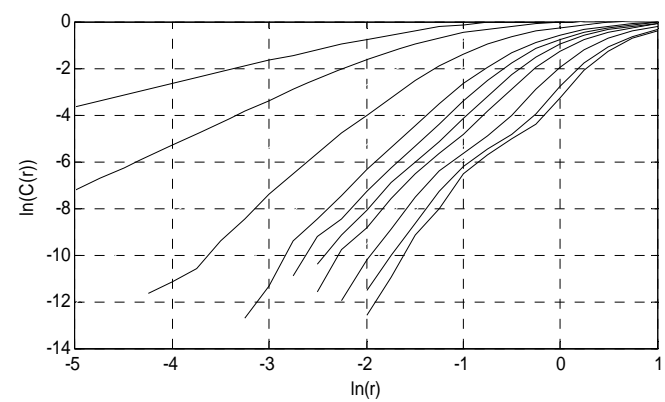

(a)Inr-InC(r) diagram

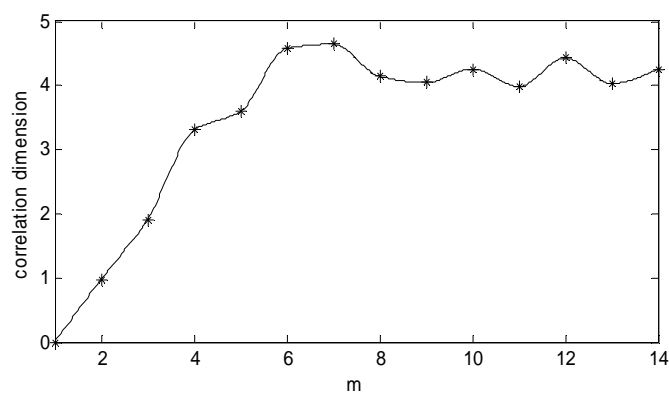

(b) Correlation dimension $\mathrm{D}(\mathrm{m})$ curve with $\mathrm{m}$

Figure 3. G-P method seeking correlation dimension and best embedding dimension 


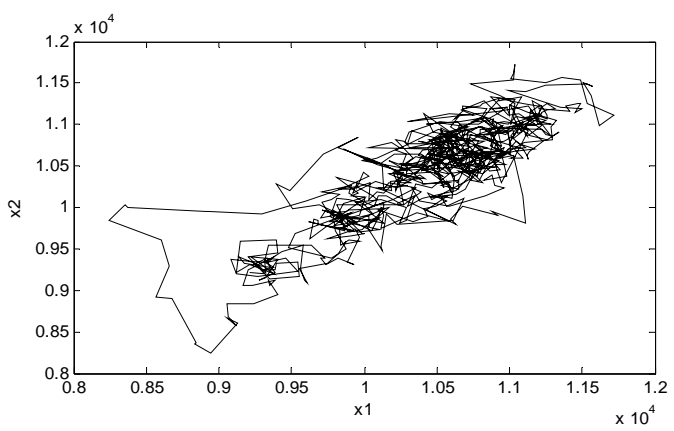

(a)Two dimension map

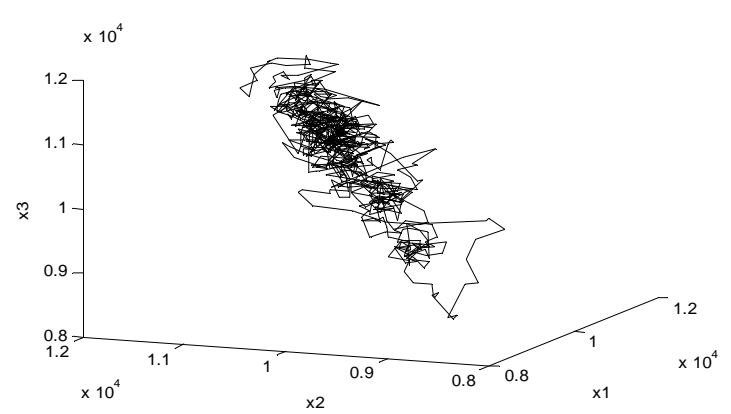

(b) Three dimension map

Figure 4. The results of phase space reconstruction

\section{B. Long-term variation of chaos characteristic in stock market}

In the above, we have analyzed time series taken from Jan, 1999 to May, 2002. However, we are still very curious about what characteristic the stock market play in a large time scale. In this part, we take time series from 1983 to 2008 for analysis. To calculate and present the variation of chaos characteristic, especially two important parameters-correlation dimension and the largest Lyapunov exponent, we choose a sets of continuous data as a group to calculate these two parameters, and we call the length of the continuous data---time window. Then we gradually move the time window forward, until to the end of the time series. In order to ensure the parameters change

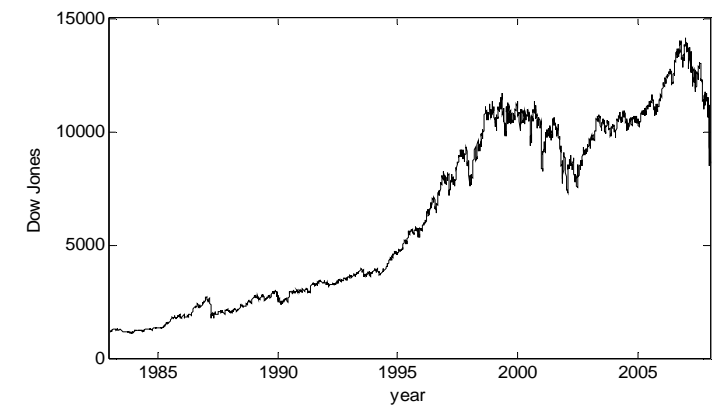

Figure 5. Time seires of Dow Jones from 1983 to 2008

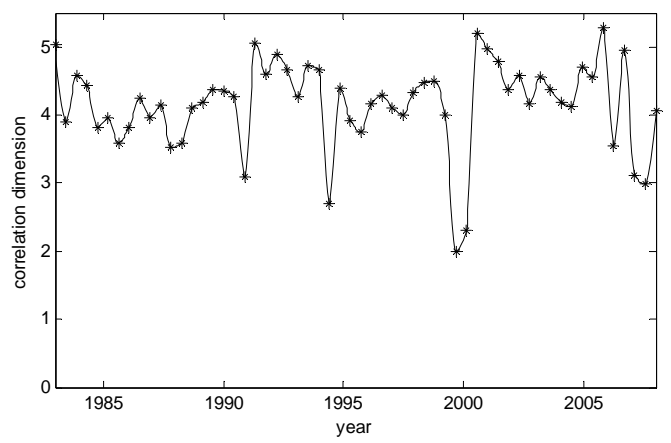

(a)time window $=500$,step $=150$ smoothly, each step we move forward should be as short as possible, preferably not more than half of the time window. However, the time window cannot be too wide, because once the selected time window is too wide, the value will reflect the entire range, not the exact value of a point. Also, the time window can not be too narrow, because if the data is too small, it will seriously affect the accuracy of the algorithm. So we must keep a balance between the wide and the narrow window. With the algorithm we used above and the moving of time window, we can get the picture of how the largest Lyapunov exponent varies in long-term, as shown in Fig. 6 and how correlation dimension varies, as shown in Fig.7.

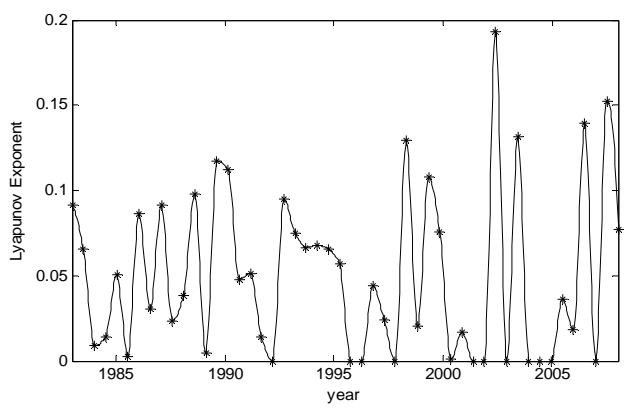

Figure 6. Variation of Lyapunov exponent (window=500, step=150)

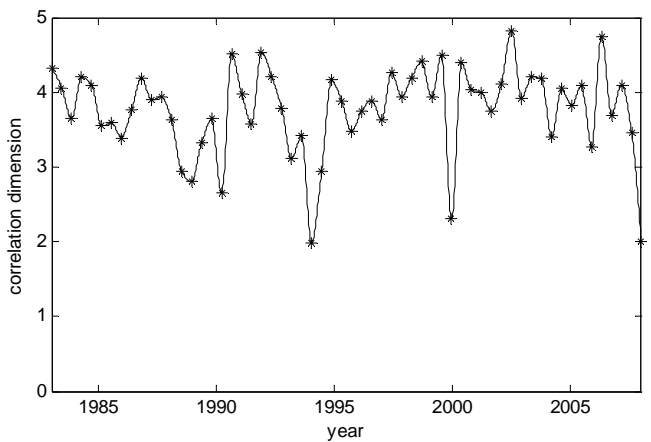

(b)time window $=200, \mathrm{step}=80$

Figure 7. Variation of Correlation Dimension 


\section{Chaos characteristic in stock market fluctuation difference}

In the above discussion, we have identified the existence of chaos in stock market time series itself. However, in fact, even though there are some fluctuations in stock market, these fluctuation can not be over half of itself in two days. Therefore, we are very curious about whether the fluctuation difference itself is chaos or random.

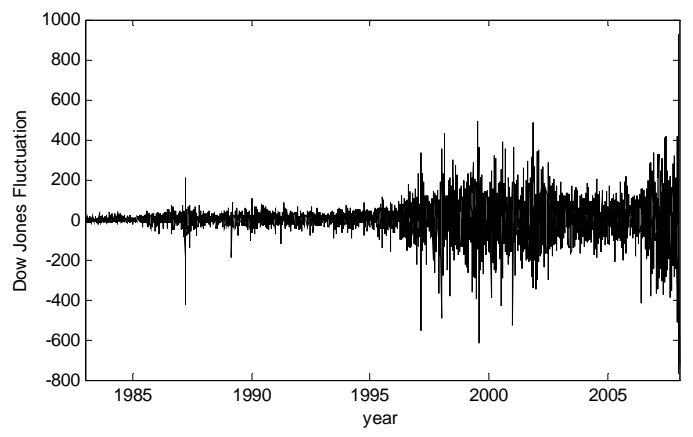

Figure 8. Time seires of Dow Jones Fluctuation

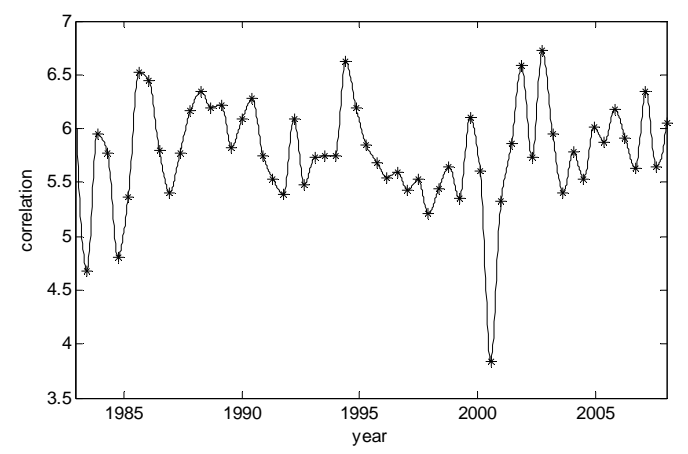

(a)time window $=500, \mathrm{step}=150$
By subtracting the data in adjacent two days, we can get the time series of stock market fluctuation difference, as shown in Fig.8. Also, these data come from Dow Jones indexes in 1983 to 2008.With the moving of time window, we can also get the picture of how the largest Lyapunov exponent varies in market fluctuation time series, as shown in Fig.9 and how correlation dimension varies, as shown in Fig.10.

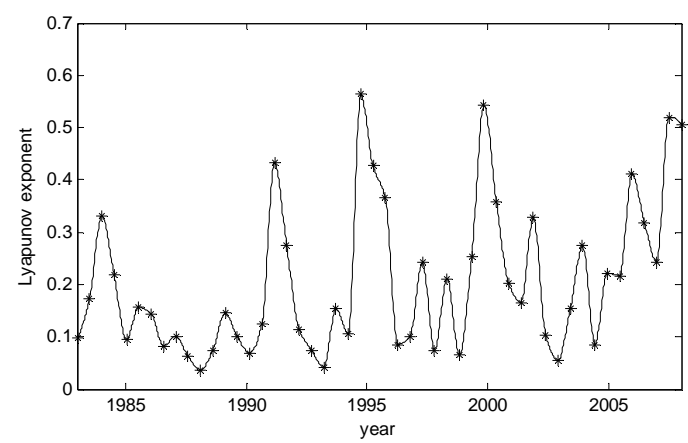

Figure 9. Variation of Lyapunov exponent(window=500, step=150)

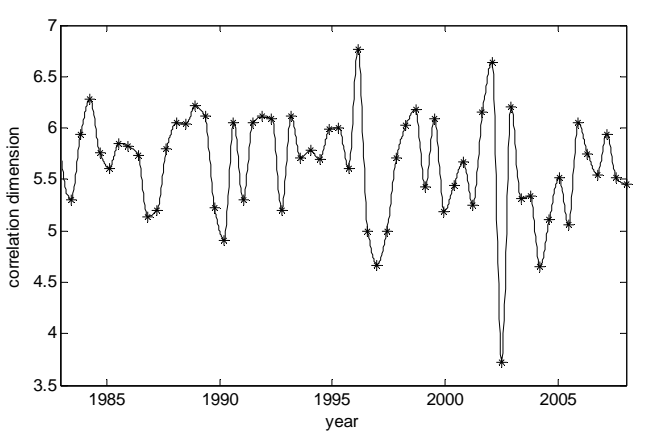

(b)time window $=200$, step $=80$

Figure 10. Variation of Correlation Dimension of stock market fluctuation

\section{DISCUSSION}

In the study we find that both the time series of Dow Jones itself and the time series of Dow Jones fluctuation difference from 1983 to 2008 shows a typical characteristic of chaos, of which the largest Lyapunov exponent is positive and the correlation dimension is fraction, which may indicating the existence of chaos. In the time series of Dow Jones itself, the correlation dimension ranges from 2 to 5, and in most of the time, the value is around 4.5. Also, in the time series of Dow Jones fluctuation difference, the correlation dimension ranges from 4 to 7, and in most of the time, the value is around 5.5,a higher dimension than itself. The higher dimension is easy to understand, because when we introduce a subtraction in the second time series, we will add a new factor to influence the space structure.

However, for the variation of the correlation dimension of Dow Jones indexes, as shown in Fig.7, there are two interesting phenomenons in it. Firstly, it seems but have not be proved that there is a quasi-periodical variation in it, and this period is about 5 years. Secondly, in the variation of the time series, the correlation dimension has several brief but rapid drops in it. As we know, the value of correlation dimension always means the complexity of the system and can reflect the number of independent variables that are necessary to describe the dynamics of the systems. Many researchers have studied in many different field ${ }^{[11-12]}$ that, for a complex nonlinear system, the reduction of correlation dimension always implies some anomalies conditions in the system, and these implies are always unfavorable. As the correlation dimension decreasing and the structure of system becoming simple, the regularity and predictability of the system will also greatly reduce. In this situation, the behavior of the system is more likely to be impact by single variable.

In both Fig. 7 and Fig.10 we can see a rapid correlation dimension drop in the year 2000. As time window has length itself, the true time for these points should be about 
one year delay than shown on the figure. So after adds one year delay, the true time should be 2001 or more for this point. It is very nature for us to guess that the drop of correlation dimension might have some relation with the Sept 11 attacks in 2001.Or in other word, the rapid drop of correlation dimension might be a reflection of the change of internal market. Again, we can find that after recovering to normal dimension for about 7 years the correlation drop rapidly again in 2008 like a cycle, which may probably indicates the internal changes of the market before financial crisis. However, these are all assumption we have made, which still need more studies to support. But the fact that there are chaos exist in stock market and the idea of using the variation of chaos indicators such as correlation, to reflect the internal changes of the market will be available in our future research.

\section{CONCLUSION}

In the paper, we find the that there are chaos characteristics exist in stock market. The index of chaos characteristic---correlation dimension, also changes through the time. We also find that correlation dimension has a quasi-periodical variation. As correlation dimension always has a important meaning for a nonlinear system, we guess that using the variation of correlation dimension can reflect some internal changes in stock market. Even though we have not given much evidence to prove this, but this idea provides a new approach for our future research in the characteristic of stock market.

\section{ACKNOWLEDGMENT}

This work is supported in part by National Natural Science Foundation of China(60574079); Zhejiang province science and technology plan by the funded projects (2011 c23097); Zhejiang fund projects (LY12F03023); Zhejiang provincial projects(Y20107866)

\section{REFERENCES}

[1] Stutzer, Michael J., "Chaotic dynamics and bifurcation in a macro model," Journal of Economic Dynamics and Control, Elsevier, 1980,2(1):353-376,

[2] Chen Ping,W.A.Barnett, Economic Theory as a Generator of Measurable Attractors, Laws of Nature and Human Conduct, Specifities and Unifying Themes, Discoveries 1985 Symposium, Brussels, I. Prigogine and D. Sanglier eds., Task Force of Research Information and Study on Science, Brussels (1987), pp. 209-224.

[3] Hesieh D, Testing foe nonlinear dynamics in foreign exchange rate[J]Journal Business,1989, 62:339-359.

[4] D.A.Hsiesh, Chaos and nonlinear dynamics application to financial market[J], Journal of Finance, 1991, 46:1839-1877.

[5] Liangsheng Chen, On the Chaotic Dynamics Analysis of China Stock Marke. The 9th International Conference for Young Computer Scientists, 2008, pp.3011-3015.

[6] Packard N H, Crutchfisld J P, Farmer J D, et al. Geometry from a time series[J]. Physical Review Letters, 1980, 45: 712-716.

[7] Takens F. Determining strange attractors in turbulence[J]. Lecture Notes in Mathmematics. Berlin: Springer, 1981, 898: 366-381.

[8] P.Grassberger, I.Procaccia, Measuring the strangeness of strange attractor[J], Physics D, 1983,9:189-208.

[9] P.Grassberger,I.Procaccia,Characterization of strange attractors, Physical Review Letter[J].1983, 50 : 346-349.

[10] Alan Wolf, Jack B Swift, Harry L swinney, et al. Detemining lyapunov exponents from a time series[J]. Physica D, 1985, 16(3): 285-317.

[11] S. Janjarasjitt, M. S. Scher, K. A. Loparo,Nonlinear dynamical analysis of the neonatal EEG time series: The relationship between sleep state and complexity [J]. Clinical Neurophysiology - CLIN NEUROPHYSIOL , 2008, 119(8): 1812-1823.

[12] Jaeseung Jeong, Nonlinear dynamics of EEG in Alzheimer's disease[J]. Drug Development Research - DRUG DEVELOP RES ,2002,56,(2):57-66. 